\title{
Solar Mass-Varying Neutrino Oscillations
}

\author{
V. Barger, ${ }^{1}$ Patrick Huber, ${ }^{1}$ and Danny Marfatia ${ }^{2}$ \\ ${ }^{1}$ Department of Physics, University of Wisconsin, Madison, Wisconsin 53706, USA \\ ${ }^{2}$ Department of Physics and Astronomy, University of Kansas, Lawrence, Kansas 66045, USA
}

(Received 23 February 2005; revised manuscript received 28 September 2005; published 15 November 2005)

We propose that the solar neutrino deficit may be due to oscillations of mass-varying neutrinos (MaVaNs). This scenario elucidates solar neutrino data beautifully while remaining comfortably compatible with atmospheric neutrino and $\mathrm{K} 2 \mathrm{~K}$ data and with reactor antineutrino data at short and long baselines (from $\mathrm{CHOOZ}$ and KamLAND). We find that the survival probability of solar MaVaNs is independent of how the suppression of neutrino mass caused by the acceleron-matter couplings varies with density. Measurements of $\mathrm{MeV}$ and lower energy solar neutrinos will provide a rigorous test of the idea.

DOI: 10.1103/PhysRevLett.95.211802

PACS numbers: $13.15 .+\mathrm{g}, 14.60 . \mathrm{Pq}, 14.60 . \mathrm{St}$

Introduction. - Neutrino oscillation experiments have conclusively demonstrated that neutrinos have mass. Also, evidence has mounted that the expansion of our Universe is in an accelerating phase caused by a negative pressure component called dark energy. While these two seemingly disparate advances are unequivocally among the most important of the last few years, our knowledge of both is woefully incomplete.

Solar neutrino data provide the only evidence of matter effects on neutrino oscillations. The large mixing angle (LMA) solution, while favored by reactor antineutrino data, is somewhat discrepant with solar data, thus calling into question how well neutrino-matter interactions are understood. It is crucial that our understanding of neutrino-matter interactions be confirmed or modified.

Dark energy is troubling because the acceleration of the Universe is a very recent phenomenon in its expansion history. This "cosmic coincidence" problem can be expressed as follows: Why are the dark matter and dark energy densities comparable today even though their ratio scales as $\sim 1 / a^{3}$ (where $a$ is the scale factor)?

The coincidence that the scale of dark energy $(2 \times$ $\left.10^{-3} \mathrm{eV}\right)^{4}$ is similar to the scale of neutrino mass-squared differences $(0.01 \mathrm{eV})^{2}$ was exploited recently in Refs. [1,2] to solve the coincidence problem. The authors of Ref. [2] considered the possibility of coupling neutrinos to dark energy by supposing that the dark energy density is a function of neutrino mass and imposing the condition that the total energy density of neutrinos and dark energy remain stationary under variations in neutrino mass. Then neutrino masses vary in such a way that the neutrino energy density and the dark energy density are related over a wide range of $a$.

A way to make the dark energy density neutrino-mass dependent is to introduce a Yukawa coupling between a sterile neutrino and a light scalar field (similar to quintessence) called the acceleron. At energy scales below the sterile neutrino mass, the effective potential of the acceleron at late times receives a contribution equal to $m_{\nu} n_{\nu}$, where $m_{\nu}$ and $n_{\nu}$ are the active neutrino mass and number density, respectively. Supersymmetric models of neutrino dark energy have been constructed [3].

Model-independent tests of neutrino dark energy are cosmological $[2,4]$. A strict relationship between the dark energy equation of state and neutrino mass is predicted. Further, since neutrino masses are predicted to scale with redshift approximately as $(1+z)^{-3}$ in the nonrelativistic regime, cosmological and terrestrial probes of neutrino mass could give conflicting results. If the acceleron couples both to neutrinos and matter, it may be possible to investigate this scenario through neutrino oscillations [5]. The coupling to matter is model dependent. The effective neutrino mass in matter is altered by the interactions via the scalar which in turn modifies neutrino oscillations.

For environments of approximately constant matter density, a satisfactory approach is to parameterize the effects of the nonstandard interactions by effective masses and mixings in the medium [5]. However, for solar neutrino oscillations it is not possible to account for the exotic matter effects by a constant shift in the oscillation parameters because the matter density in the Sun varies by several orders of magnitude.

In this Letter we investigate solar mass-varying neutrino $(\mathrm{MaVaN})$ oscillations; these have not been studied previously. We will show that since the neutrinos propagate adiabatically, the specific dependence of the evolving masses on the acceleron potential is irrelevant, so the predicted survival probability depends only on the masses at their production sites. We then demonstrate how MaVaNs improve the agreement with solar neutrino data while being perfectly consistent with KamLAND data [6]. Finally, we illustrate via a calculation of the survival probabilities of atmospheric muon neutrinos crossing the earth's core that the scheme is consistent with atmospheric neutrino data. Since we focus on astrophysical and terrestrial neutrinos, the dependence of the neutrino mass on redshift is not pertinent to our considerations.

Effect of acceleron interactions on neutrino masses. - At low redshifts, the contribution to the neutrino mass caused by the interactions of the acceleron with electrons and 
neutrinos can be written as

$$
M_{i}=\lambda_{\nu_{i}}\left[\lambda_{e} n_{e}+\sum_{i} \lambda_{\nu_{i}}\left(n_{\nu_{i}}^{C \nu B}+\frac{m_{\nu_{i}}}{E_{\nu_{i}}} n_{\nu_{i}}^{\mathrm{rel}}\right)\right] / m_{\phi}^{2}
$$

where $\lambda_{\nu_{i}}\left(\lambda_{e}\right)$ is the Yukawa coupling of the acceleron to $\nu_{i}$ (the electron). Throughout, when we quote values for $\lambda$, we mean $|\lambda|$. In principle, the scalar $\phi$ has a mass, $m_{\phi}$, that depends on $n_{e}$ and the $n_{\nu_{i}}$. This dependence is weak since the underlying assumption in obtaining Eq. (1) is that $\phi$ does not fluctuate significantly from its background value in the current epoch. The number density of the cosmic neutrino background in one generation of neutrinos and antineutrinos is $n_{\nu_{i}}^{C \nu B} \sim 112 \mathrm{~cm}^{-3} \sim 10^{-12} \mathrm{eV}^{3}$, the number density of relativistic neutrinos in the background frame is $n_{\nu_{i}}^{\text {rel }}$, and the electron number density is $n_{e}$. Here, $m_{\nu_{i}}$ are neutrino masses in a background dominated environment. We assume the heaviest $\nu_{i}$ to be $\mathcal{O}(0.05) \mathrm{eV}$ in the present epoch, and that as a result of their nonnegligible velocities, the neutrino overdensity in the Milky Way from gravitational clustering can be neglected [7]. Then, $m_{\nu_{i}}$ essentially represent the masses of terrestrial neutrinos in laboratory experiments like those measuring tritium beta decay. Since the neutrinos under consideration are light, we do not expect the instabilities of highly nonrelativistic neutrino dark energy [8].

In principle, we should include a nucleon-acceleron Yukawa coupling. Since the electron-acceleron and nucleon-acceleron couplings are arbitrary (within bounds from gravitational tests), we can parameterize their combined effect on $M_{i}$ through $\lambda_{e}$, although this is not rigorously true for two reasons: (1) Conventional matter effects [9] for active neutrino oscillations do not depend on the nucleon number density $n_{N}$. (2) The $n_{e}$ and $n_{N}$ distributions in the Sun do not have the same shape [10]. Nonetheless, this simplification suffices for our purposes.

Tests of the gravitational inverse square law require the coupling of a scalar to the square of the gluon field strength to be smaller than $0.01 m_{N} / M_{P l} \sim 10^{-21}$ [11], where $m_{N}$ is the nucleon mass. Since we have chosen to embody the effects of the couplings of the acceleron to the nucleons and electrons in $\lambda_{e}$, the latter bound applies to $\lambda_{e}$. In the region of the solar core where $p p$ neutrinos are produced, $n_{e}^{0} \sim 60 N_{A} / \mathrm{cm}^{3} \sim 10^{11} \mathrm{eV}^{3}$ [10]. (Here and henceforth, we denote the electron number density at the point of neutrino production by $n_{e}^{0}$ ). Thus, for $\lambda_{e}$ close to its upper bound, $\lambda_{e} n_{e}^{0} \sim 10^{-10} \mathrm{eV}^{3}$.

The cosmic neutrino background contributes negligibly to the mass shift even for $\lambda_{\nu_{i}}$ of $\mathcal{O}(1)$. The $p p$ reaction creates neutrinos with the highest number density in the production region $\left(\sim 7 \times 10^{-8} \mathrm{eV}^{3}\right)$ and lowest energies $\left(E_{\nu} \sim 0.3 \mathrm{MeV}\right)$ of all other processes in the $p p$ chain and CNO cycle. Thus, $p p$ neutrinos have the highest possible $m_{\nu} n_{\nu}^{\text {rel }} / E_{\nu}$, which, for $m_{\nu}$ of $\mathcal{O}(1) \mathrm{eV}$, is at most $n_{\nu_{i}}^{C \nu B}$. In sum, the dominant contribution to the mass shift at the creation point arises from the $\lambda_{e} n_{e}$ term.
We require that some $M_{i}$ be $\mathcal{O}\left(10^{-3}-10^{-2}\right) \mathrm{eV}$ at neutrino production in the Sun. Then, for an assumed $m_{\phi}^{2}$ of $\mathcal{O}\left(10^{-11}\right) \mathrm{eV}^{2}$, we need $\lambda_{\nu_{i}} \sim 10^{-4}-10^{-3}$. For this range of $\lambda_{\nu_{i}}$, the cosmic neutrino contribution in Eq. (1) is 5 to 6 orders of magnitude smaller than the electron contribution, and the $p p$ neutrino contribution is 8 to 9 orders of magnitude smaller. The $C \nu B$ density becomes dominant only after $n_{e}$ drops by about 6 orders of magnitude. This does not happen until neutrinos reach the surface of the Sun. As the neutrinos leave the Sun, $m_{\nu}$ approaches its background value. The choice $\lambda_{\nu_{i}} \sim 10^{-3}$ serves more than one purpose. In addition to fixing the maximum values of $M_{i}$, it ensures that $n_{\nu_{i}}^{C \nu B}$ can be neglected for the entire path of the neutrinos through the Sun.

Solar MaVaN oscillations. - In the framework of the standard model (SM) with massive neutrinos and conventional neutrino-matter interactions, solar (atmospheric) neutrinos oscillate with $\delta m_{s}^{2} \sim 8 \times 10^{-5} \mathrm{eV}^{2}$ and $\theta_{s} \sim$ $\pi / 6\left(\left|\delta m_{a}^{2}\right| \sim 0.002 \mathrm{eV}^{2}\right.$ and $\theta_{a} \sim \pi / 4$ [6]). In our notation, $\delta m_{s}^{2}\left(\delta m_{a}^{2}\right)$ is the solar (atmospheric) mass-squared difference and $\theta_{s}, \theta_{a}$, and $\theta_{x}$ are the mixing angles conventionally denoted by $\theta_{12}, \theta_{23}$, and $\theta_{13}$, respectively [6]. We also know that solar and atmospheric neutrino oscillations largely occur independently of each other because $\theta_{x}$ must be small from the nonobservance of $\bar{\nu}_{e} \rightarrow \bar{\nu}_{\mu}$ oscillations at the atmospheric scale. In fact, data from the $\mathrm{CHOOZ}$ experiment demand $\sin ^{2} \theta_{x} \lesssim 0.05$ at the $2 \sigma$ C.L. in the conventional picture.

With the additional freedom that the $M_{i}$ provide, there is no reason to believe that the three neutrino oscillation dynamics factorizes into the dynamics of two two-neutrino subsystems. Nevertheless, since our purpose here is to show that MaVaN oscillations are consistent with solar and atmospheric neutrino data while obeying the $\mathrm{CHOOZ}$ bound, we are entitled to accomplish our goal via construction. A simplifying assumption is that the decoupling of solar and atmospheric neutrino oscillations continues to hold for MaVaNs. Then, the CHOOZ bound is automatically satisfied and we need to demonstrate that the two-neutrino framework is adequate for both anomalies.

The evolution equations for solar MaVaN oscillations in the two-neutrino framework are

$$
\begin{aligned}
i \frac{d}{d r}\left(\begin{array}{c}
\nu_{e} \\
\nu_{\mu}
\end{array}\right)= & \frac{1}{2 E_{\nu}}\left[U\left(\begin{array}{cc}
{\left[m_{1}-M_{1}(r)\right]^{2}} & M_{3}(r)^{2} \\
M_{3}(r)^{2} & {\left[m_{2}-M_{2}(r)\right]^{2}}
\end{array}\right)\right. \\
& \left.\times U^{\dagger}+\left(\begin{array}{cc}
A(r) & 0 \\
0 & 0
\end{array}\right)\right]\left(\begin{array}{c}
\nu_{e} \\
\nu_{\mu}
\end{array}\right) .
\end{aligned}
$$

Here, $M_{i}$ is a linear combination of those in Eq. (1), $U$ is the usual $2 \times 2$ mixing matrix, $E_{\nu}$ is the neutrino energy, and $A(r)=2 \sqrt{2} G_{F} n_{e}(r) E_{\nu}=1.52 \times$ $10^{-7} \mathrm{eV}^{2} n_{e}(r) E_{\nu}(\mathrm{MeV})$ is the amplitude for $\nu_{e}-e$ forward scattering in matter with $n_{e}$ in units of $N_{A} / \mathrm{cm}^{3}$. For typical ${ }^{8} \mathrm{~B}$ neutrinos $\left(E_{\nu} \sim 7 \mathrm{MeV}\right) n_{e}^{0} \simeq 100$, for ${ }^{7} \mathrm{Be}$ neutrinos $\left(E_{\nu} \sim 0.9 \mathrm{MeV}\right) n_{e}^{0} \simeq 90$, and for $p p$ neutrinos $\left(E_{\nu} \sim 0.3 \mathrm{MeV}\right) n_{e}^{0} \simeq 60$. The matter term $A^{0}$ at the points 
of origin is about $10^{-4} \mathrm{eV}^{2}, 10^{-5} \mathrm{eV}^{2}$, and $10^{-6} \mathrm{eV}^{2}$ for ${ }^{8} \mathrm{~B},{ }^{7} \mathrm{Be}$, and $p p$ neutrinos, respectively. With our choice of $\left|M_{i}^{2}\right|$ of $\mathcal{O}\left(10^{-5}-10^{-4}\right) \mathrm{eV}^{2}$ at neutrino production, we expect nonstandard matter effects to be of the same order as standard matter effects.

We adopt a matter dependence of the form $M_{i}(r)=$ $\mu_{i}\left[n_{e}(r) / n_{e}^{0}\right]^{k}$, where $k$ parameterizes the dependence of the neutrino mass on $n_{e}$, and $\mu_{i}$ is the neutrino mass shift at the point of neutrino production. As noted above, we expect $k$ to be close to unity, but we shall show that a wider range of $k$ is allowed by oscillation data. We have implicitly made the approximation that all neutrinos are created with the same values of $\mu_{i}$ irrespective of where in the Sun they are produced. Since almost all solar neutrinos are produced within $r<0.2 r_{\odot}$, for which $n_{e}$ falls by about a factor of 3 from its value at the center of the Sun, we consider the approximation to be reasonable.

We make the parameter choices $\mu_{1}=m_{1}=0, \mu_{2}=$ $0.0077 \mathrm{eV}, \mu_{3}=i 0.0022 \mathrm{eV}, m_{2}=0.0089 \mathrm{eV}$, and $\theta=$ 0.62 . The value of $\delta m^{2}$ in a background dominated environment is $m_{2}^{2}=7.9 \times 10^{-5} \mathrm{eV}^{2}$. We will refer to $\mu_{i}$ as MaVaN parameters and $m_{i}$ as background parameters. As we show, this set of parameters is consistent with KamLAND data and improves the agreement with solar data.

The evolution of the mass eigenstates as they travel through the Sun is governed by

$$
4 i E_{\nu} \frac{d}{d r}\left(\begin{array}{l}
\nu_{1} \\
\nu_{2}
\end{array}\right)=\left(\begin{array}{cc}
-\Delta(r) & -4 i E_{\nu} d \theta_{m} / d r \\
4 i E_{\nu} d \theta_{m} / d r & \Delta(r)
\end{array}\right)\left(\begin{array}{l}
\nu_{1} \\
\nu_{2}
\end{array}\right),
$$

where $\Delta(r)$ is the magnitude of the mass-squared difference of the eigenvalues of the matrix in square brackets in Eq. (2) and $\theta_{m}$ is the effective mixing angle in matter. The value of $\theta_{m}$ at the creation point of the neutrino is

$$
\cos 2 \theta_{m}^{0}=\frac{-B-2 \mu_{3}^{2} \sin 2 \theta}{\sqrt{B^{2}+\left(m_{2}-\mu_{2}\right)^{4} \sin ^{2} 2 \theta+4 \mu_{3}^{2}\left(A^{0} \sin 2 \theta+\mu_{3}^{2}\right)}},
$$

where $B=A^{0}-\left(m_{2}-\mu_{2}\right)^{2} \cos 2 \theta$, which yields the standard result in the limit that $\mu_{2}, \mu_{3} \rightarrow 0$. The condition for adiabatic evolution [12] is $\frac{\Delta(r)}{4 E_{\nu}\left|d \theta_{m} / d r\right|} \equiv Q(r) \gg 1$.

In Fig. 1, we show how $\theta_{m}$ and $Q^{-1}$ depend on $r / r_{\odot}$ for $E_{\nu}=0.1,0.74,5 \mathrm{MeV}$ with $k=1$. We do not show the evolution of $\Delta$ since it is smooth throughout. Notice the step in $\theta_{m}$ at $r / r_{\odot} \sim 0.0035$ for $E_{\nu}=0.74 \mathrm{MeV}$. (The energy at which this occurs depends on the background and MaVaN parameters chosen). The step manifests itself as a large spike in $Q^{-1} ; Q<10$ only in a $1 \mathrm{keV}$ spread around $0.74 \mathrm{MeV}$. While adiabaticity is violently violated in this narrow range of energy, it is undetectable because experimental resolutions are much larger than $1 \mathrm{keV}$.

For all practical purposes, the evolution is adiabatic and the survival probability is given by the standard formula [13], $P\left(\nu_{e} \rightarrow \nu_{e}\right)=\left(1+\cos 2 \theta_{m}^{0} \cos 2 \theta\right) / 2$, with $\cos 2 \theta_{m}^{0}$ from Eq. (3). Thus, we find that the survival probability
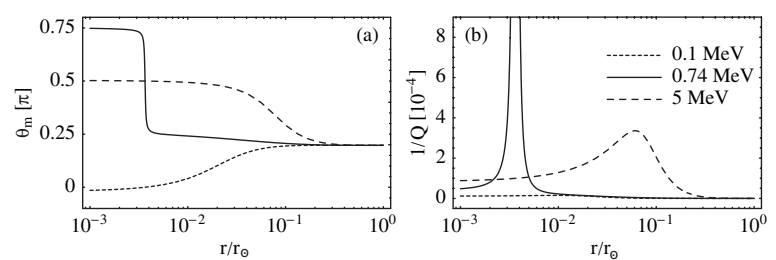

FIG. 1. (a) $\theta_{m}$ and (b) $Q^{-1}$ as a function of $r / r_{\odot}$ for three representative energies. The adiabatic condition $Q \gg 1$ is violated at $r / r_{\odot} \sim 0.0035$ because $d \theta_{m} / d r$ becomes very large.

of solar neutrinos is independent of $k$ so long as the neutrinos propagate adiabatically. The dependence on the acceleron-matter couplings enters only at the production point of the neutrino via the $\mu_{i}$.

MaVaN oscillations vs data.-We now compare the predictions of this framework with solar data. To this end, we use the recently extracted average survival probabilities of the low energy $(p p)$, intermediate energy $\left({ }^{7} \mathrm{Be}\right.$, pep, ${ }^{15} \mathrm{O}$, and $\left.{ }^{13} \mathrm{~N}\right)$, and high energy $\left({ }^{8} \mathrm{~B}\right.$ and hep $)$ neutrinos; for details see Ref. [14]. From Fig. 2, we see that the MaVaN survival probability almost passes through the central values of the three data points. The agreement with intermediate energy data is remarkably improved compared to the LMA solution because $P_{\mathrm{MaVaN}}\left(\nu_{e} \rightarrow \nu_{e}\right)$ approaches $\sin ^{2} \theta$ for lower $E_{\nu}$ than for $P_{\mathrm{SM}}\left(\nu_{e} \rightarrow \nu_{e}\right)$. For the same solution, it is possible for $p p$ neutrinos to have a higher survival probability than the vacuum value, $1-$ $0.5 \sin ^{2} 2 \theta$. This control over the width of the transition region and the larger difference between the survival probabilities of the $p p$ and ${ }^{8} \mathrm{~B}$ neutrinos (than $\cos ^{2} \theta \cos 2 \theta$ for the LMA solution) is a result of the freedom provided by the additional free parameter $\mu_{3}$. Keeping in mind that the survival probability of the neutrinos incident on Earth is independent of $k$, if we set $\mu_{3}=k=0$, we recover the standard MSW case with $m_{2}$ replaced by $m_{2}-\mu_{2}$. The $k$ dependence reappears for neutrinos traversing the Earth.

An important question is whether MaVaN oscillations are consistent with KamLAND data. The solid curve in Fig. 3(a) is $d P\left(\bar{\nu}_{e} \rightarrow \bar{\nu}_{e}\right) \equiv P_{\mathrm{SM}}\left(\bar{\nu}_{e} \rightarrow \bar{\nu}_{e}\right)-$ $P_{\mathrm{MaVaN}}\left(\bar{\nu}_{e} \rightarrow \bar{\nu}_{e}\right)$, for a mean KamLAND baseline of $180 \mathrm{~km}$ and energy resolution $7.3 \% / \sqrt{E(\mathrm{MeV})}$. Here, $P_{\mathrm{SM}}$ is calculated for $k=1 / 2$ with $\delta m^{2}=8 \times 10^{-5} \mathrm{eV}^{2}$ and $\theta=0.55$; the latter are the vacuum parameters favored

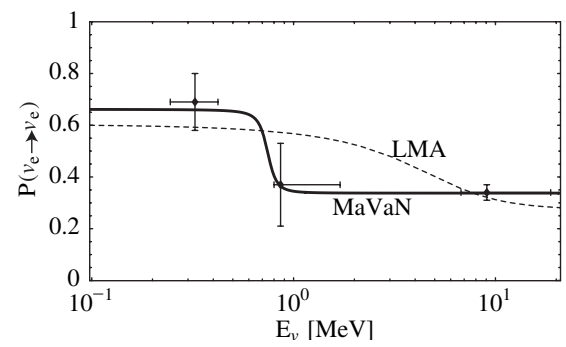

FIG. 2. $P\left(\nu_{e} \rightarrow \nu_{e}\right)$ vs $E_{\nu}$ for MaVaN oscillations (solid curve). The dashed curve corresponds to conventional oscillations with the best-fit solution to KamLAND data. 


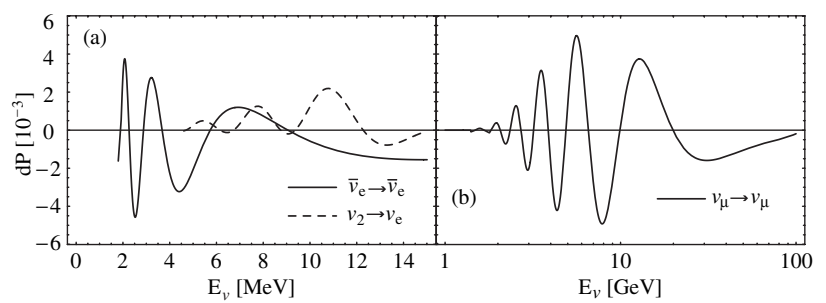

FIG. 3. (a) $d P$ for reactor $\bar{\nu}_{e}$ incident at KamLAND (solid curve), and $d P\left(\nu_{2} \rightarrow \nu_{e}\right)$ for solar neutrinos passing through the center of the Earth (dashed curve). (b) $d P\left(\nu_{\mu} \rightarrow \nu_{\mu}\right)$ for atmospheric neutrinos passing through the Earth's core.

by KamLAND data. Any $k>1 / 2$ produces a $d P$ that is acceptable.

A relevant question is if Earth matter effects are substantial for solar MaVaNs. Since $\nu_{e}$ with energy above a few $\mathrm{MeV}$ exit the Sun and arrive at the Earth in the intermediate neutrino mass eigenstate (denoted by $\nu_{2}$ ), it is appropriate to study $\nu_{2} \rightarrow \nu_{e}$ transitions to assess the size of these effects. The dashed curve in Fig. 3(a) is the energy-averaged $d P\left(\nu_{2} \rightarrow \nu_{e}\right)$ (assuming a $10 \%$ energy resolution) for neutrinos passing through the center of the Earth in which case matter effects are expected to be enhanced. $P_{\mathrm{MaVaN}}\left(\nu_{2} \rightarrow \nu_{e}\right)$ deviates only slightly from the usual oscillations and is in accord with a tiny day-night effect as required by Super-Kamiokande and SNO data.

We next show in Fig. 3(b) that atmospheric neutrino data are also consistent with two-neutrino $\nu_{\mu} \rightarrow \nu_{\tau}$ oscillations for MaVaN parameters of comparable size to those in the solar sector $\left(k=1 / 2, \mu_{2}^{a}=0.01 \mathrm{eV}, \mu_{3}^{a}=0.003 \mathrm{eV}\right.$, and $\left.m_{3}=0.047 \mathrm{eV}\right)$. Here, $P_{\mathrm{SM}}$ is calculated for $\delta m^{2}=$ $0.0021 \mathrm{eV}^{2}$ and $\theta=\pi / 4 . d P\left(\nu_{\mu} \rightarrow \nu_{\mu}\right)$ is averaged over the earth's core $\left(\cos \theta_{Z}=0.8-1\right.$, where $\theta_{Z}$ is the nadir angle), for a $10 \%$ energy resolution. We also confirm that $d P\left(\nu_{\mu} \rightarrow \nu_{\mu}\right)$ at the $\mathrm{K} 2 \mathrm{~K}$ baseline is well below experimental sensitivity.

Conclusions. - We have shown that oscillations of variable mass neutrinos (that result in exotic matter effects of the same size as standard matter effects) lead to an improved agreement (relative to conventional oscillations) with solar neutrino data while remaining compatible with KamLAND, CHOOZ, K2K, and atmospheric data.

MaVaN oscillations are perfectly compatible with solar data because the survival probability can change from a higher-than-vacuum value (at low energies) to $\sin ^{2} \theta$ (at high energies) over a very narrow range of energies. Since the neutrino propagation is highly adiabatic, the survival probability of solar neutrinos is independent of $k$.

Whether or not an explanation of solar neutrino data requires MaVaN oscillations will be answered by experiments that will measure the survival probability of $\mathrm{MeV}$ and lower energy neutrinos. As shown in Ref. [15], other tests in reactor and long-baseline experiments emerge when the scheme presented here is embedded in a comprehensive model that can explain all extant neutrino oscillation data including the LSND anomaly and a future MiniBooNE result.

We have considered neutrinos with background mass of $\mathcal{O}(0.01) \mathrm{eV}$. For such light neutrinos, only modeldependent (neutrino oscillation) tests of the MaVaN scenario are viable because the model-independent (cosmological) tests become inoperable. There are two reasons for this: (1) The dark energy behaves almost exactly as a cosmological constant today. (2) If these light neutrinos do not cluster sufficiently, the local neutrino mass is the same as the background value, which is below the sensitivity of tritium beta-decay experiments. Then, highredshift cosmological data (which should show no evidence for neutrino mass) and data from tritium beta-decay experiments will be consistent.

We thank R. Garisto, J. Learned, S. Pakvasa, A. Smirnov, G. Steigman, and K. Whisnant for helpful comments. We have made use of the GLoBES software [16]. This research was supported by the DOE under Grant No. DE-FG02-95ER40896, by the NSF under Grant No. EPS-0236913, by the State of Kansas through KTEC, and by the WARF.

[1] P. Q. Hung, hep-ph/0010126; P. Gu, X. Wang, and X. Zhang, Phys. Rev. D 68, 087301 (2003).

[2] R. Fardon, A. Nelson, and N. Weiner, J. Cosmol. Astropart. Phys. 10 (2004) 005.

[3] R. Takahashi and M. Tanimoto, hep-ph/0507142; R. Fardon, A. Nelson, and N. Weiner, hep-ph/0507235.

[4] R. D. Peccei, Phys. Rev. D 71, 023527 (2005).

[5] D. Kaplan, A. Nelson, and N. Weiner, Phys. Rev. Lett. 93, 091801 (2004); K. M. Zurek, J. Cosmol. Astropart. Phys. 10 (2004) 058.

[6] For a recent review see V. Barger, D. Marfatia, and K. Whisnant, Int. J. Mod. Phys. E 12, 569 (2003).

[7] A. Ringwald and Y. Wong, J. Cosmol. Astropart. Phys. 12 (2004) 005.

[8] N. Afshordi, M. Zaldarriaga, and K. Kohri, Phys. Rev. D 72, 065024 (2005).

[9] L. Wolfenstein, Phys. Rev. D 17, 2369 (1978); S. Mikheev and A. Yu. Smirnov, Sov. J. Nucl. Phys. 42, 913 (1985); V. Barger et al., Phys. Rev. D 22, 2718 (1980).

[10] J. Bahcall and R. Ulrich, Rev. Mod. Phys. 60, 297 (1988).

[11] E. Adelberger, B. Heckel, and A. Nelson, Annu. Rev. Nucl. Part. Sci. 53, 77 (2003).

[12] H. Bethe, Phys. Rev. Lett. 56, 1305 (1986); V. Barger, R. Phillips, and K. Whisnant, Phys. Rev. D 34, 980 (1986).

[13] S. J. Parke, Phys. Rev. Lett. 57, 1275 (1986).

[14] V. Barger et al., Phys. Lett. B 617, 78 (2005); Phys. Rev. Lett. 88, 011302 (2002); Phys. Lett. B 509, 19 (2001).

[15] V. Barger, D. Marfatia, and K. Whisnant, hep-ph/0509163.

[16] P. Huber, M. Lindner, and W. Winter, Comput. Phys. Commun. 167, 195 (2005). 